\title{
Programme du DESC d'Andrologie 1996
}

\section{LUNDI 19 FEVRIER}

$10 \mathrm{~h}-12 \mathrm{~h}$

$14 \mathrm{~h}-16 \mathrm{~h}$

$16 \mathrm{~h}-18 \mathrm{~h}$

$18 \mathrm{~h}-19 \mathrm{~h}$
Pr. CZYBA

Pr. NAVRATIL

Dr. DECOURT

A. SPIRA
- Sexualité et ontogenèse

- Courbures de verge et maladie de La Peyronie

- Développement psychosexuel

- Epidémiologie des comportements sexuels

MARDI 20 FEVRIER

$9 \mathrm{~h}-12 \mathrm{~h}$

Dr. GIULIANO

$14 \mathrm{~h}-15 \mathrm{~h}$

$16 \mathrm{~h}-18 \mathrm{~h}$

17h-18h

Dr. ROLLET

Dr. CABANIS

Pr. NAVRATIL

Dr. CABANIS
- Anatomo-physiologie de l'érection, de l'éjaculation et de l'orgasme

- Libido et ses perturbations

- Interrogatoire et évaluation d'un trouble sexuel chez l'homme

- Orgasme et dysorgasmie

\section{MERCREDI 21 FEVRIER}

$\begin{array}{lll}9 \mathrm{~h}-11 \mathrm{~h} & \text { Dr. GIULIANO } & \text { - Méthodes d'explorations de l'érection } \\ 11 \mathrm{~h}-12 \mathrm{~h} & \text { Pr. JARDIN } & \begin{array}{l}\text { - Dysfonctions sexuelles organiques } \\ \text { (et iatrogènes) }\end{array}\end{array}$

14h-16h

$16 \mathrm{~h}-18 \mathrm{~h}$

Dr. PENOCHET

- Névroses et sexualité

- Perversions sexuelles

\section{JEUDI 22 FEVRIER}

$9 \mathrm{~h}-12 \mathrm{~h}$

R. TREMBLAY

- Sexualité de l'enfant et de l'adolescent.

- Information sexuelle.

- Sexualité et vieillissement

$14 \mathrm{~h}-16 \mathrm{H}$

B. QUATELLAS

- MST-Sida-Toxicomanie et Sexualité

$16 \mathrm{~h}-18 \mathrm{~h}$

Dr. DOLIVEAU

- Affections dermato-vénérologiques de la verge

VENDREDI 23 FEVRIER

$9 \mathrm{~h}-12 \mathrm{~h}$

Dr. CHEVRET

$14 \mathrm{~h}-15 \mathrm{H}$

Dr. FOREST

$15 \mathrm{~h}-17 \mathrm{~h}$

Dr. BONDIL

$17 \mathrm{~h}-18 \mathrm{~h}$

Pr. SULTAN
- Dysfonctions sexuelles psychologiques

- La femme de l'impuissant et de l'éjaculateur précoce

- Micropénis

- Les allongements de la verge

- Priapismes

- Conduite à tenir devant une ambiguité sexuelle à la naissance 


\section{LUNDI 3 JUIN}

10h-12H Dr. BONIERBALE - Introduction aux différents modes de thérapie des troubles sexuels

14h-16h Dr. GRAFEILLE - Le transsexualisme

Pr. LERICHE

16h-18h Dr. BRENOT - Sexualité et Phylogénèse

\section{MARDI 4 JUIN}

$9 \mathrm{~h}-10 \mathrm{~h}$

Dr. ASSABGUI

- Apport de l'hypnose, de la relaxation et de la musicothérapie aux prises en charge sexologiques

$10 \mathrm{~h}-11 \mathrm{~h}$

M. BACCIGALUPO - Bases de la sexo-analyse et utilisation des fantasmes en thérapie

$11 \mathrm{~h}-12 \mathrm{~h}$

A. DURANDAU - L'identité sexuelle

$14 \mathrm{~h}-15 \mathrm{H}$

A. DURANDAU

- Les troubles de l'orientation sexuelle

$15 \mathrm{~h}-16 \mathrm{~h}$

Dr. DESBARRATS

- Délinquance sexuelle

$16 \mathrm{~h}-17 \mathrm{~h}$

Dr. BONAL

- Thérapie corporelle en sexologie

$17 \mathrm{~h}-18 \mathrm{~h}$

Dr. BONAL

- Cancer et sexualité

\section{MERCREDI 5 JUIN}

$\begin{array}{lll}\text { 9h-11h } & \text { Dr. BENGOZHI } & \text { - Psychopathologie du couple } \\ \text { 11h-12h } & \text { Pr. CZYBA } & \text { - Stérilité et sexualité } \\ \text { 14h-15h } & \text { Pr. CZYBA } & \text { - Sexualité et PMA } \\ \text { 15h-16h } & \text { Dr. BALMES } & \text { - Evénements de vie et sexualité } \\ \text { 16h-18h } & \text { Pr. COSTA } & \text { - Handicap et sexualité }\end{array}$

\section{JEUDI 6 JUIN}

$9 \mathrm{~h}-12 \mathrm{~h}$

Dr. BENGOZHI - Psychothérapie du couple

$14 \mathrm{~h}-16 \mathrm{~h}$

Pr. SARRAMON

- Traitement chirurgical de l'impuissance

$16 \mathrm{~h}-17 \mathrm{~h}$

Pr. PONTONNIER

- Ethique médicale et thérapies sexuelles

\section{VENDREDI 7 JUIN}

$9 \mathrm{~h}-12 \mathrm{~h}$

Pr. COSTA

Dr. COLSON

$14 \mathrm{~h}-16 \mathrm{~h}$

\section{MONTRELAY}

- Traitement médicamenteux de l'impuissance

- Les injections intracaverneuses

- La puissance masculine

- Approche psycho-analytique. 